\title{
Sleep laboratory test referrals in Canada: Sleep Apnea Rapid Response Survey
}

\author{
Jessica Evans MSc MD(c) ${ }^{1}$, Robert Skomro MD², Helen Driver $\mathrm{PhD}^{3}$, Brian Graham $\mathrm{PhD}^{4}$, Irvin Mayers $\mathrm{MD}^{5}$, \\ Louise McRae $\mathrm{MSc}^{1}$, Joseph Reisman $\mathrm{MD}^{6}$, Corneliu Rusu $\mathrm{MSc}^{1}$, Teresa $\mathrm{To} \mathrm{PhD}^{7}$, John Fleetham $\mathrm{MD}^{8}$
}

\begin{abstract}
J Evans, R Skomro, H Driver, et al. Sleep laboratory test referrals in Canada: Sleep Apnea Rapid Response survey. Can Respir J 2014;21(1):e4-e10.
\end{abstract}

BACKGROUND: An estimated 5.4 million Canadian adults have been diagnosed with sleep apnea or are at high risk of experiencing obstructive sleep apnea (OSA). There are no recent Canadian data regarding access to and predictors of referral for diagnostic testing in these populations.

METHODS: The Sleep Apnea Rapid Response survey sampled 8647 Canadian adults and captured information about risk, testing, diagnosis and treatment of sleep apnea. Predictors of sleep laboratory test referrals were assessed using log-linked binomial regression modelling. Information regarding sleep testing facilities was updated at the provincial and regional levels. RESULTS: Approximately $76.8 \%$ (95\% CI $70.1 \%$ to $83.6 \%$ ) of adult Canadians with sleep apnea and 5.1\% (95\% CI 3.4\% to 6.7\%) of those at high risk for OSA reported being referred to a sleep laboratory. Significant predictors of sleep laboratory referral in the general population were male sex, middle age, overweight or obese, a chronic condition, having a regular medical doctor and reporting symptoms of sleep apnea. Region of residence was also a predictor of reported sleep laboratory referral, with individuals from Ontario being more likely to report being referred to a sleep laboratory versus individuals from other regions.

CONCLUSION: Individuals reporting risk factors and symptoms associated with OSA were more likely to report a sleep laboratory testing referral compared with those without risk factors or symptoms. However, Canada's diagnostic sleep laboratory testing capacity varies across regions and is believed to be inadequate given the number of individuals at high risk for OSA who did not report testing referral.

Key Words: Diagnostic testing referral; Sleep apnea; Sleep laboratory testing capacity

$\mathrm{O}$ structive sleep apnea (OSA) is a common chronic condition; its prevalence, estimated to be $3 \%$ to $7 \%$ among adult males and $2 \%$ to $5 \%$ among adult females in Western countries, along with the prevalence of its risk factors, such as obesity and aging, are increasing (1). A recent Public Health Agency of Canada (PHAC) survey indicated that 22\% (5.4 million) of adult Canadians report either being diagnosed with sleep apnea (3\%) or are at high risk for OSA (19\%) (2). The exact proportion of high risk for OSA cases that are true cases of OSA remains unknown; however, if one conservatively assumes that only $15 \%$ of the high-risk cases have OSA (3\% of the overall population), in addition to the $3 \%$ who report being diagnosed with sleep apnea, the prevalence of OSA approaches that of reported diagnosed diabetes (6\%) (3).

With the growing awareness of OSA and its associated outcomes, such as increased risk of systemic hypertension (4), atherosclerosis and cardiovascular events (5), and motor vehicle collisions (6) and, with evidence demonstrating the effectiveness of therapy (7-8), there has

\section{Les aiguillages vers des tests en laboratoire du sommeil au Canada : sondage sur la réponse rapide à l'apnée du sommeil}

HISTORIQUE : On estime que 5,4 millions d'adultes canadiens ont un
diagnostic d'apnée du sommeil ou sont très vulnérables à une apnée obstruc-
tive du sommeil (AOS). Aucunes données canadiennes récentes ne portent
sur l'accès aux tests diagnostiques et sur les prédicteurs d'aiguillage vers ces
tests au sein de ces populations. MÉTHODOLOGIE : Le sondage sur la réponse rapide à l'apnée du sommeil a sondé 8647 adultes canadiens et permis de saisir de l'information sur le risque, les tests, le diagnostic et le traitement de l'apnée du sommeil. Les chercheurs ont évalué les prédicteurs d'aiguillage vers des tests en laboratoire du sommeil au moyen de la modélisation de régression binomiale liée au logarithme. L'information sur les laboratoires de tests du sommeil ont été mises à jour sur les scènes provinciale et régionale.

RÉSULTATS : Environ 76,8 \% (95 \% IC 70,1 \% à 83,6 \%) des adultes canadiens ayant de l'apnée du sommeil et 5,1\% (95 \% IC 3,4 \% à 6,7 \%) de ceux très vulnérables à une $\mathrm{AOS}$ ont déclaré avoir été aiguillés vers un laboratoire du sommeil. Les prédicteurs significatifs d'aiguillage vers un laboratoire du sommeil dans la population générale étaient le sexe masculin, l'âge mûr, l'embonpoint ou l'obésité, une maladie chronique, le fait d'avoir un médecin traitant et des symptômes déclarés d'apnée du sommeil. La région de résidence était également un prédicteur d'aiguillage déclaré vers un laboratoire du sommeil, les habitants de l'Ontario étant plus susceptibles de recevoir un tel aiguillage que ceux des autres régions.

CONCLUSION : Les personnes qui déclarent des facteurs de risque et des symptômes associés à l'AOS étaient plus susceptibles de déclarer un aiguillage vers des tests en laboratoire du sommeil que celles qui ne présentaient pas de facteurs de risque ou de symptômes. Cependant, la capacité des tests diagnostiques en laboratoire du sommeil au Canada varie selon les régions et semble insuffisante compte tenu du nombre de personnes très vulnérables à l'AOS qui n'ont pas déclaré avoir été aiguillés pour des tests.

${ }^{1}$ Centre for Centre for Chronic Disease Prevention, Public Health Agency of Canada, Ottawa, Ontario; ${ }^{2}$ Department of Medicine, University of

Saskatchewan, Saskatoon, Saskatchewan; 3Sleep Disorders Laboratory, Kingston General Hospital and Department of Medicine, Queen's

University, Kingston, Ontario; ${ }^{4}$ Division of Respirology, Department of Medicine, University of Saskatchewan, Saskatoon, Saskatchewan;

${ }^{5}$ Department of Medicine, University of Alberta, Edmonton, Alberta; ${ }^{6}$ Department of Paediatrics, University of Ottawa, Ottawa; ${ }^{7}$ Child

Health Evaluative Sciences, The Hospital for Sick Children, Toronto, Ontario; ${ }^{8}$ Department of Medicine, University of British Columbia,

Vancouver, British Columbia

Correspondence: Ms Jessica Evans, Centre for Chronic Disease Prevention, Public Health Agency of Canada, 785 Carling Avenue, Ottawa,

Ontario K1A 0K9. E-mail jessica.evans@mail.mcgill.ca 
laboratory beds per capita in Canada; and to examine the predictors of sleep laboratory testing in the adult Canadian population.

\section{METHODS}

\section{The Sleep Apnea Rapid Response, 2009 Canadian Community} Health Survey

The Sleep Apnea Rapid Response (SARR) questionnaire was developed and funded by PHAC, and was conducted by Statistics Canada as a component of the 2009 Canadian Community Health Survey (CCHS). The CCHS is a cross-sectional survey that annually samples 65,000 respondents $\geq 12$ years of age and provides estimates at the health region level. Residents of Indian reserves, Crown lands, institutions and the Territories, as well as full-time members of the Canadian Forces are not included. The CCHS uses three sampling frames to select the sample of households and generate a sample representative of the Canadian population. The area frame is a complex, two-stage stratified design in which each stratum is formed of clusters. The clusters are selected using a sampling method with a probability proportional to size; the final sample is generated by a systematic sampling of dwellings stratified according to health regions within the clusters. In addition, random household telephone numbers were selected using a list frame of telephone numbers and a random digit dialling sampling frame that complemented the area frame and that were also stratified according to health region. The selection of a household member was made at the time of contact for data collection. Household members $\geq 12$ years of age were listed and chosen using various selection probabilities based on age and household composition. During January and February 2009 (the sampling frame of the SARR module), a total sample of 9523 individuals was obtained, with an overall response rate of $73 \%$. The weighted sample represents approximately $98 \%$ of the Canadian population. Due to sample size limitations associated with reported diagnosed sleep apnea among individuals 12 to 17 years of age, the current study focused on adults $\geq 18$ years of age $(n=8647)$.

\section{Analysis}

The analyses were performed using SAS Enterprise Guide version 4.1 (SAS, USA). Estimates were weighted to the Canadian population by applying sampling weights that accounted for the unequal probability of being selected into the survey. SDs and 95\% CIs were estimated using the bootstrap technique (13). The bootstrap method allows for variance estimation while accounting for the complex multistage survey design. The process involves drawing repeated random samples, with replacement, from the observations to obtain a set of estimates. Estimates associated with a coefficient of variation $>33.3$ or a cell size $<10$ were not reported due to high sampling variability. Statistical significance was defined at $\mathrm{P}<0.05$.

Potential predictors of reported sleep laboratory test referral were identified a priori. Bivariate log-linked binomial modelling was used to assess the relationship between the variables of interest and reported sleep laboratory test referral; prevalence rate ratios (PRR) were subsequently estimated. All variables found to be significant in the bivariate regression model, as well as all confounders were included in the final adjusted multivariable log-linked binomial regression models. Potential confounders were identified as such if they resulted in a $10 \%$ change in the relative risk for the association between the exposure and outcome (14). Potential effect modification was explored by including multiplicative interaction terms into the model; however, none of the interactions assessed were significant. Variance inflation factors were also assessed, and multicollinearity was not observed in any of the models (variance inflation factor <10) (15).

\section{Defining select covariates}

Individuals with self-reported diagnosed sleep apnea were identified as those who responded 'yes' to the following question: 'Have you been told by a health professional that you have sleep apnea?' Individuals with self-reported sleep laboratory testing referral were identified as those who responded 'yes' to the following question: 'Have you ever been referred to a sleep lab for overnight testing?' Individuals at high risk for OSA, excluding those who reported diagnosed sleep apnea, were identified using the STOP tool, which screens patients for OSA based on Snoring, Tiredness, Observed apneas and high blood Pressure (16). Given specific length and time guidelines for the CCHS Rapid Response modules, which dictated restrictions on the number and length of questions, the use of the STOP tool for estimating risk of OSA was a logistical decision.

\section{Sleep laboratory testing capacity data}

Data regarding numbers of sleep laboratories and numbers of sleep laboratory beds for each province was provided by a Canadian Sleep Society (CSS) database, which included data from provincial regulatory bodies and home care companies. Confirmation of the data was performed by telephone survey conducted in late 2010 and early 2011, and was additionally verified by members of the CSS and the Canadian Thoracic Society. Facilities that only performed home-based sleep testing with portable monitors (ie, level 3 testing) were excluded from the list.

\section{RESULTS}

\section{Population characteristics}

Population distributions according to various characteristics are presented in Table 1 for the total population and for individuals reporting having been referred to a sleep laboratory. The proportion of individuals who reported a sleep laboratory test referral was similar among the total population (4.8\%) and those at high risk for OSA (5.1\%) (Table 2). Nearly one in four individuals with diagnosed sleep apnea had not been referred to a sleep laboratory for overnight testing.

\section{Predictors of referral for overnight sleep laboratory testing}

The prevalence of reported referral to a sleep laboratory according to various population characteristics and the associated rate ratios are presented in Table 3 and Figure 1.

In the adjusted model, significant predictors of reported sleep laboratory test referral were body mass index (BMI), number of chronic conditions, regular medical doctor, age, sex and region (Figure 1). Individuals who were male, 45 to 64 years of age, were overweight or obese, had one or more of the five chronic conditions of interest, had a regular medical doctor and resided in Ontario were more likely to report sleep laboratory test referral.

The PRRs of sleep laboratory test referral for symptoms of sleep apnea, including those of the STOP tool, are reported in Table 4. Reported testing referral was greater among individuals reporting symptoms of sleep apnea - loud snoring, tiredness and observed apnea - compared with those not reporting these symptoms. Observed apnea was the strongest symptom predictor for sleep laboratory test referral. The proportion of individuals at high risk for OSA who were referred for sleep laboratory testing was nearly two times greater than in those who were not at high risk for OSA.

\section{Chronic conditions and sleep laboratory test referral}

The prevalence of high risk for OSA as well as the proportion reporting sleep laboratory test referral according to chronic condition is presented in Table 5. Compared with the prevalence of high risk of OSA (19.0\% [ $95 \%$ CI $17.6 \%$ to $20.4 \%]$ ) and the proportion reporting sleep laboratory test referral $(4.8 \%$ [95\% CI $4.1 \%$ to $5.5 \%])$ in the general population, the prevalence of high risk for OSA and proportion reporting sleep laboratory test referrals was significantly higher for individuals with chronic conditions, except for migraine headaches (Table 5).

The prevalence of high risk for OSA was greatest among individuals with hypertension, chronic obstructive pulmonary disease (COPD), heart disease and diabetes, while the largest proportion reporting a sleep laboratory test was among respondents with COPD, diabetes, heart disease, and mood or anxiety disorder. Reporting sleep 
TABLE 1

Study population characteristics

\begin{tabular}{|c|c|c|}
\hline \multirow[b]{2}{*}{ Population characteristic } & \multicolumn{2}{|c|}{$\begin{array}{l}\text { Proportion reporting various } \\
\text { population characteristics* }\end{array}$} \\
\hline & $\begin{array}{c}\text { Total } \\
\text { population } \\
(n=8647) \\
\end{array}$ & $\begin{array}{l}\text { Individuals report- } \\
\text { ing sleep lab test- } \\
\text { ing referral }(n=422)\end{array}$ \\
\hline \multicolumn{3}{|l|}{ Sex } \\
\hline Male & $49.1(48.8-49.4)$ & $60.5(53.5-67.5)$ \\
\hline \multicolumn{3}{|l|}{ Age, years } \\
\hline $18-44$ & $47.7(47.4-47.9)$ & $28.0(21.4-34.7)$ \\
\hline $45-64$ & $35.6(35.4-35.8)$ & $53.7(46.7-60.7)$ \\
\hline$\geq 65$ & $16.7(16.7-16.8)$ & $18.3(13.9-22.6)$ \\
\hline \multicolumn{3}{|l|}{ Ethnicity } \\
\hline White & $80.6(78.8-82.4)$ & $86.7(81.6-91.8)$ \\
\hline \multicolumn{3}{|l|}{ Body mass index, $\mathrm{kg} / \mathrm{m}^{2}$} \\
\hline$<25$ & $48.2(46.4-50.0)$ & $17.4(11.6-23.2)^{\ddagger}$ \\
\hline$\geq 25$ to $<30$ & $34.2(32.6-35.9)$ & $46.8(39.0-54.6)$ \\
\hline$\geq 30$ to $<35$ & $13.2(12.0-14.4)$ & $22.0(16.3-27.6)$ \\
\hline$\geq 35$ & $4.4(3.9-5.1)$ & $13.9(9.1-18.7)^{\ddagger}$ \\
\hline \multicolumn{3}{|l|}{ Smoking status } \\
\hline Never smoker & $37.1(35.3-38.9)$ & $27.1(20.3-33.9)$ \\
\hline Former smoker & $41.8(40.1-43.4)$ & $54.2(46.5-61.8)$ \\
\hline Current smoker & $21.1(19.7-22.6)$ & $18.7(13.6-23.9)$ \\
\hline \multicolumn{3}{|l|}{ Marital status } \\
\hline Ever married/common-law & $77.1(75.9-78.3)$ & $86.2(80.7-91.6)$ \\
\hline \multicolumn{3}{|l|}{ Educational attainment } \\
\hline$<$ Secondary school & $8.4(7.6-9.2)$ & $6.1(2.6-9.7)^{\ddagger}$ \\
\hline Secondary school graduation & $16.0(14.7-17.4)$ & $14.7(9.4 \mathrm{v} 20.0)^{\ddagger}$ \\
\hline Postsecondary school graduation & $75.6(74.0-77.2)$ & $79.2(73.1-85.3)$ \\
\hline \multicolumn{3}{|l|}{ Total annual household income, $\$$} \\
\hline$<30,000$ & $14.5(13.3-15.7)$ & $15.4(11.2-19.5)$ \\
\hline 30,000 to 99,999 & $47.4(45.7-49.2)$ & $52.7(45.5-59.9)$ \\
\hline$\geq 100,000$ & $24.6(23.0-26.2)$ & $32.0(24.5-39.4)$ \\
\hline Not stated & $13.5(12.3-14.8)$ & $11.9(5.2-18.6)^{\ddagger}$ \\
\hline \multicolumn{3}{|l|}{ Chronic conditions $^{\dagger}$} \\
\hline 0 of 5 conditions & $70.1(68.5-71.7)$ & $47.9(39.7-56.0)$ \\
\hline $1-2$ of 5 conditions & $27.9(26.4-29.4)$ & $45.0(37.5-52.5)$ \\
\hline $3-5$ of 5 conditions & $2.0(1.6-2.3)$ & $7.2(4.4-9.9)^{\ddagger}$ \\
\hline
\end{tabular}

Medical doctor

Has a regular medical doctor $\quad 84.6$ (83.3-85.9) 95.9 (93.2-98.5)

Data presented as \% $(95 \% \mathrm{Cl})$. *Estimates weighted to the Canadian population; IIncludes hypertension, heart disease, diabetes, stroke and mood disorder; \#Interpret with caution - estimate associated with high sampling variability. lab Laboratory

laboratory testing among individuals with hypertension was relatively low given the large proportion at high risk for OSA in this group. The proportion reporting a test referral did not differ significantly according to chronic condition.

Geography and sleep laboratory testing referral

Geographical region region was a strong predictor of reported sleep laboratory test referrals (Table 3 ). The prevalence according to geographical region of reported diagnosed sleep apnea, high risk for OSA and the proportion reporting sleep laboratory test referrals are presented in Table 6. After adjustment for confounders, the proportion reporting sleep laboratory referral was significantly less in the Prairie region, Quebec and the Atlantic regions compared with Ontario. The nonsignificance of the adjusted PRR for British Columbia was potentially due to limited sample power, as indicted by the degree of variability associated with the estimate as well as the strength of the association represented by the unadjusted and adjusted point PRR estimate.
TABLE 2

Proportion reporting sleep laboratory testing referral among adults $\geq 18$ years of age

\begin{tabular}{lc}
\hline & $\begin{array}{c}\text { Proportion reporting sleep } \\
\text { laboratory testing referral*, } \\
\%(95 \% \mathrm{Cl})\end{array}$ \\
\hline Total Canadian population & $4.8(4.1-5.5)$ \\
Individuals with reported diagnosed sleep apnea & $76.8(70.1-83.6)$ \\
Individuals at high risk for OSA & $5.1(3.4-6.7)$ \\
Individuals not reporting diagnosed sleep & $1.4(0.9-1.8)$ \\
apnea and not at high risk for OSA & \\
\hline
\end{tabular}

*Estimates weighted to the Canadian population; ${ }^{+}$High risk for obstructive sleep apnea (OSA) excludes individuals who reported diagnosed sleep apnea and is defined by the STOP tool (see text)

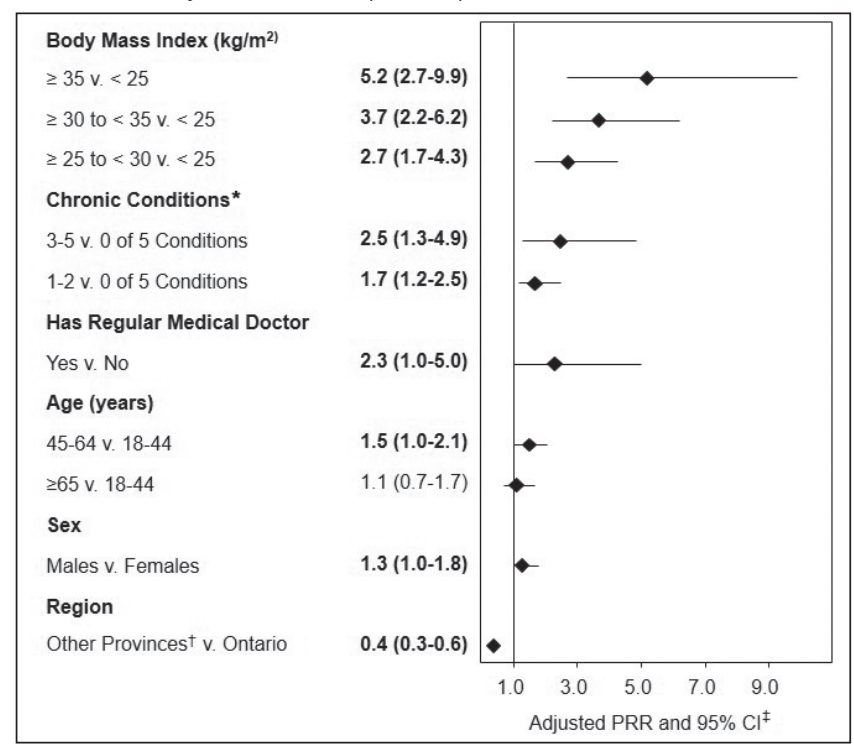

Figure 1) Adjusted prevalence rate ratios (PRR) for reported sleep laboratory testing referrals among adults $\geq 18$ years of age and older. Estimates are weighted to the Canadian population; "Includes hypertension, heart disease, diabetes, stroke and mood disorder; ${ }^{\dagger}$ Excludes the Territories; ${ }^{\ddagger}$ Adjusted for sex, age, ethnicity, body mass index, smoking, marital status, educational attainment, household income, chronic conditions, having a regular medical doctor and region. Bolded values indicate $P<0.05 . v$ Versus

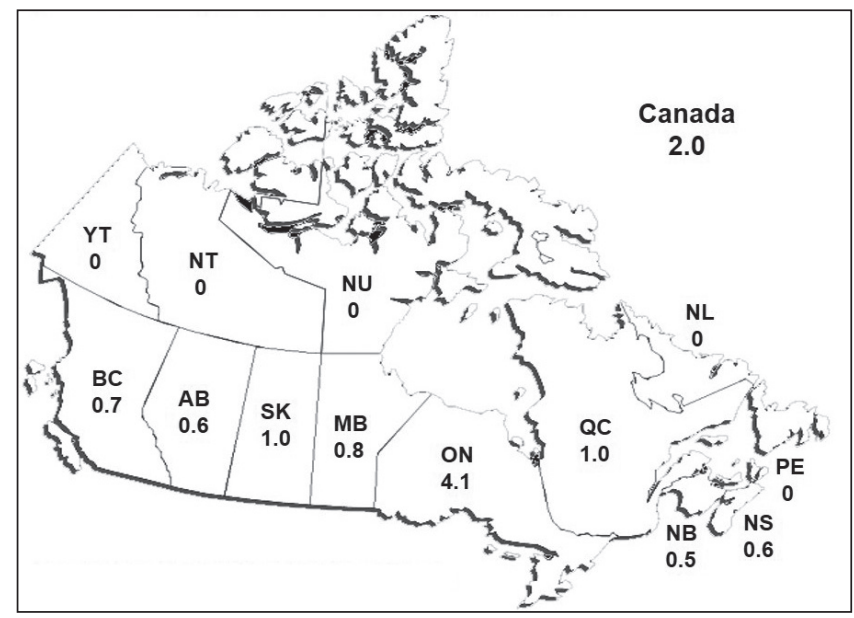

Figure 2) Number of sleep testing beds per 100,000 population in Canada, 2010. Estimates do not include level 3 or pediatric testing facilities. $A B$ Alberta; BC British Columbia; MB Manitoba; NB New Brunswick; NL Newfoundland and Labrador; NS Nova Scotia; NT Northwest Territories; NU Nunavut; ON Ontario; PE Prince Edward Island; QC Quebec; SK Saskatchewan; YT Yukon Territory 
TABLE 3

Prevalence and rate ratios for reported overnight sleep laboratory testing referrals among adults $\geq 18$ years of age

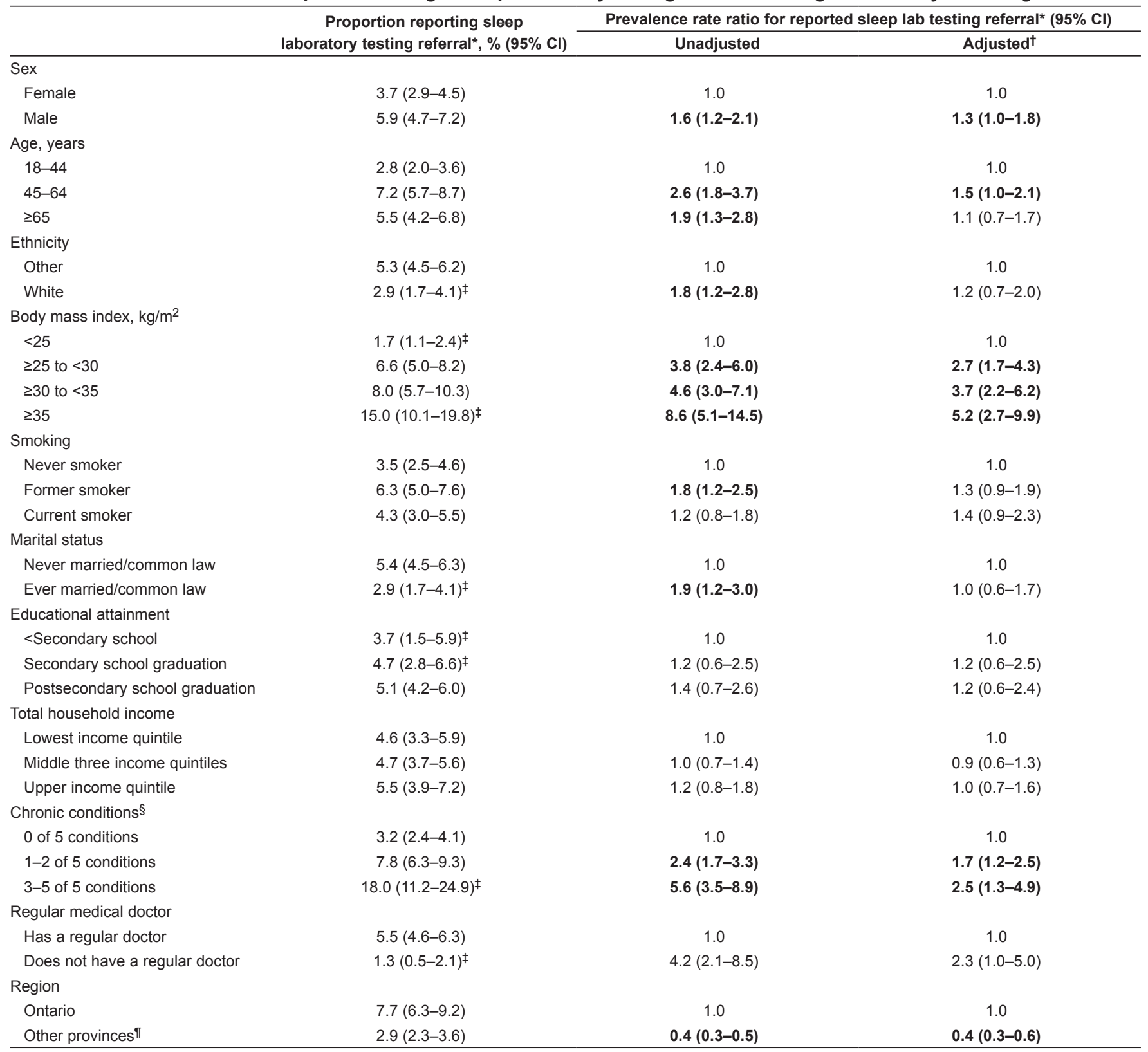

Bolded values indicate $P<0.05,1.0$ represents the reference category. ${ }^{*}$ Estimates weighted to the Canadian population; ${ }^{\dagger}$ Adjusted for sex, age, ethnicity, body mass index, smoking, marital status, educational attainment, household income, chronic conditions, having a regular medical doctor and region; $¥$ Interpret with caution, estimate associated with high sampling variability; §Includes hypertension, heart disease, diabetes, stroke and mood disorder; $\llbracket$ Excludes the Territories

Distribution of sleep laboratories across Canada

Current estimates of number of sleep laboratories and per 100,000 population sleep testing beds according to province and region are presented in Table 7 and Figure 2. The number of sleep laboratory beds per 100,000 population varies greatly across Canada, ranging from 0 in several provinces/territories to 4.1 beds per 100,000 in Ontario. The number of sleep laboratory beds in Ontario is twice the national estimate for Canada. Overall, the number of sleep laboratory beds per 100,000 population in Canada has increased to 2.0 in 2010 from the 1.4 estimated in 2004. The largest relative increase in the number of beds was observed in the Prairie region, followed by Quebec, Ontario and British Columbia. In the Atlantic region, the number of beds per 100,000 population decreased slightly, while estimates for the Territories remained at 0 . Within the regions, a high degree of provincial variability exists in the number of sleep laboratory beds per 100,000 population.

\section{DISCUSSION}

Approximately $4.8 \%$ of the total population and $76.8 \%$ of individuals with reported diagnosed sleep apnea reported having been referred to a sleep laboratory for overnight testing. Significant predictors of sleep laboratory referral included male sex, middle age, overweight, having a chronic condition, having a regular medical doctor and reporting symptoms of sleep apnea. Our data are consistent with previous findings that male sex, increased BMI and multiple comorbid chronic conditions are associated with OSA. Seventy one per cent of the individuals referred to a sleep laboratory were diagnosed with sleep apnea or were at high risk for OSA, suggesting that of the potential sleep disorders being investigated, suspicion of sleep apnea is likely the most common reason for referral to sleep laboratory.

While several sleep apnea symptoms and risk factors were associated with sleep laboratory test referral, systemic hypertension, a key clinical 


\section{TABLE 4}

Prevalence rate ratios of sleep laboratory testing referral according to symptoms of obstructive sleep apnea (OSA) among adults $\geq 18$ years of age

\begin{tabular}{|c|c|c|c|}
\hline & \multirow{2}{*}{$\begin{array}{l}\text { Proportion reporting } \\
\text { sleep laboratory testing } \\
\text { referral }{ }^{*} \%(95 \% \mathrm{Cl})\end{array}$} & \multicolumn{2}{|c|}{$\begin{array}{c}\text { Prevalence rate ratio for sleep laboratory } \\
\text { testing referral* }(95 \% \mathrm{Cl})\end{array}$} \\
\hline & & Unadjusted & Adjusted ${ }^{\dagger}$ \\
\hline Does not snore & $3.1(2.3-3.9)$ & 1.0 & 1.0 \\
\hline Snores slightly louder than heavy breathing or as loud as talking & $4.2(3.0-5.4)$ & $0.8(0.6-1.2)$ & $0.9(0.6-1.4)$ \\
\hline Snores louder than talking or loud enough to be heard through closed doors ${ }^{\ddagger}$ & $11.4(8.4-14.3)$ & $3.2(2.3-4.4)$ & $1.5(1.0-2.3)$ \\
\hline Does not often feel tired, fatigued, or sleepy during the daytime & $3.0(2.2-3.8)$ & 1.0 & 1.0 \\
\hline Often feels tired, fatigued, or sleepy during the daytime ${ }^{\ddagger}$ & $7.0(5.8-8.3)$ & $2.4(1.7-3.3)$ & $2.2(1.5-3.1)$ \\
\hline Not observed to stop breathing during sleep & $2.9(2.3-3.5)$ & 1.0 & 1.0 \\
\hline Observed to stop breathing during sleep $\ddagger$ & $26.7(21.5-31.9)$ & $9.3(7.0-12.4)$ & $6.6(4.7-9.4)$ \\
\hline Does not have hypertension & $4.0(3.1-4.8)$ & 1.0 & 1.0 \\
\hline Has or is being treated for hypertension ${ }^{\ddagger}$ & $8.3(6.4-10.2)$ & $2.1(1.5-2.9)$ & $1.3(0.9-2.0)$ \\
\hline Has difficulty falling or staying asleep none, some, or a little of the time & $4.0(6.5-11.7)$ & 1.0 & 1.0 \\
\hline Has difficulty falling or staying asleep most or all of the time & $9.1(6.5-11.7)$ & $2.3(1.6-3.2)$ & $2.0(1.4-2.8)$ \\
\hline Awakens with the feeling of gasping or choking never, rarely, or sometimes & $4.5(14.0-31.6)$ & 1.0 & 1.0 \\
\hline Awakens with the feeling of gasping or choking often or very often & $22.8(14.0-31.6)$ & $5.1(3.3-7.8)$ & $2.9(1.9-4.6)$ \\
\hline Not diagnosed with sleep apnea and not at high risk for OSA & $1.4(0.9-1.8)$ & 1.0 & 1.0 \\
\hline At high risk of OSA§ & $5.1(3.4-6.7)$ & $3.2(2.1-5.0)$ & $1.9(1.1-3.3)$ \\
\hline
\end{tabular}

Bolded values indicate $P<0.05,1.0$ represents the reference category. ${ }^{\star}$ Estimates weighted to the Canadian population; ${ }^{\dagger}$ Adjusted for sex, age, ethnicity, body mass index, smoking, marital status, educational attainment, household income, chronic conditions, regular medical doctor and region (note: ratio for hypertension not adjusted for chronic conditions due to colinearity issues); ¥Components of the STOP tool for estimating high risk for OSA (16); §High risk for OSA excludes individuals who reported diagnosed sleep apnea and is defined by the STOP tool (see text)

feature of sleep apnea, was not a significant predictor of referral. Although $60.3 \%$ of the respondents with systemic hypertension were at high risk for OSA, only $8.3 \%$ of this population reported being referred for sleep laboratory testing, which was lower than the proportion referred for testing among several other chronic disease categories (eg, COPD, diabetes, heart disease, mood or anxiety disorder). These results suggest that patients with systemic hypertension are not being sufficiently identified at risk for OSA, despite the relationship between OSA and hypertension (4). On the other hand, use of the STOP criteria could lead to an overestimation of the prevalence of high risk for OSA among individuals with hypertension, and perhaps the discrepancy between proportion referred for testing and those at high risk for OSA among those with hypertension is not as great (although likely still present) as has been estimated (16). Several provinces perform unattended portable sleep monitoring or overnight oximetry to diagnose OSA; we are aware that publicly funded portable sleep monitoring exists in at least three provinces (British Columbia, Manitoba and Saskatchewan). Our data indicate that nearly one-quarter (23.2\%) of Canadians with reported diagnosed sleep apnea were potentially diagnosed by methods other than sleep laboratory testing (76.8\% of individuals with sleep apnea had been referred to sleep laboratories). Some of these individuals may not have been tested at all. Because the sleep laboratory referral question specified 'overnight testing', we cannot exclude the possibility that some individuals may have been diagnosed with sleep apnea based on portable sleep monitoring or overnight oximetry. Moreover, our survey asked whether individuals had ever been 'referred' for a sleep test, but not if such testing had ever been performed; thus, we may have captured individuals who were still waiting to undergo a sleep laboratory test.

The CSS database of level 1 sleep laboratories was generated using provincial regulatory databases and was supplemented by national home care company lists in which level 1 laboratory activity is not regulated. Database accuracy was verified in each province by Canadian Thoracic Society and CSS members from those regions, and all laboratories on the list were called to confirm their operation and the number of beds. It is estimated that $>90 \%$ of in-laboratory (level-1) testing activity has been captured using this methodology. Conversely, unattended portable sleep monitoring (level-3 testing; excluded from the analysis) remains unregulated; it is currently
TABLE 5

Proportion reporting sleep laboratory testing referral according to chronic condition

\begin{tabular}{lcc}
\hline $\begin{array}{l}\text { Self-reported health } \\
\text { professional-diagnosed } \\
\text { chronic condition }\end{array}$ & $\begin{array}{c}\text { Proportion at high } \\
\text { risk for OSA }\end{array}$ & $\begin{array}{c}\text { Proportion reporting } \\
\text { sleep laboratory } \\
\text { testing referral }\end{array}$ \\
\hline $\begin{array}{l}\text { Mood or anxiety disorder }{ }^{\ddagger} \\
\text { Arthritis or back problems }\end{array}$ & $28.9(23.8-34.0)$ & $11.2(8.1-14.3)$ \\
$\quad$ (excluding fibromyalgia) & $29.5(26.7-32.4)$ & $7.7(6.2-9.3)$ \\
Asthma & $28.8(23.2-34.5)$ & $9.2(5.9-12.6)^{\S}$ \\
COPD (COPD, chronic & $51.0(42.4-59.7)$ & $13.1(7.8-18.3)^{\S}$ \\
$\quad$ bronchitis, or emphysema) & & \\
Diabetes & $48.0(41.2-54.8)$ & $12.1(8.6-15.7)$ \\
Heart disease & $50.0(43.4-56.5)$ & $11.9(7.1-16.6)^{\S}$ \\
Hypertension & $60.3(56.0-64.6)$ & $8.3(6.4-10.2)$ \\
Migraine headaches & $24.3(19.6-29.0)$ & $5.5(3.5-7.5)^{\S}$ \\
Urinary incontinence ${ }^{\star *}$ & $45.7(36.9-54.4)$ & $10.1(6.0-14.2)^{\S}$ \\
\hline
\end{tabular}

Data presented as \% (95\% Cl). *Estimates weighted to the Canadian population; High risk for obstructive sleep apnea (OSA) excludes individuals who reported diagnosed sleep apnea and is defined by the STOP tool; \#Includes phobia, obsessive compulsive disorder, panic disorder, depression, bipolar, mania and dysthymia; §Interpret with caution, estimate associated with high

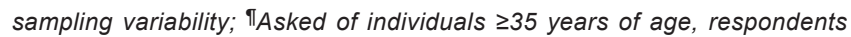
$<35$ years of age were coded as not having the condition; **Asked of individuals $\geq 25$ years, respondents $<25$ years of age were coded as not having the condition. COPD Chronic obstructive pulmonary disease

difficult to ascertain the availability of portable testing in Canada. Regional discrepancies in the number of beds per 100,000 population were observed, with estimates being four to 10 times greater in Ontario compared with other provinces where sleep laboratory beds exist. The regional differences in beds per 100,000 population estimates and, subsequently, rates of sleep laboratory test referral and diagnosed sleep apnea, may reflect different practice models and public coverage among the provinces. The potential underdiagnosis of OSA is a Canada-wide issue, as is suggested by the large differences observed between the prevalence of individuals at high risk for OSA and diagnosis of sleep apnea across all provinces. The ratio of individuals at 
TABLE 6

Prevalence and rate ratios of reported diagnosed obstructive sleep apnea (OSA) and sleep laboratory testing referral according to region among adults $\geq 18$ years of age

\begin{tabular}{|c|c|c|c|c|c|c|}
\hline & \multicolumn{4}{|c|}{ Prevalence* $^{*}(95 \% \mathrm{CI})$} & & \\
\hline & \multirow{2}{*}{$\begin{array}{c}\text { Reported sleep } \\
\text { laboratory testing } \\
\text { referral }\end{array}$} & \multirow{2}{*}{$\begin{array}{l}\text { Reported diagnosed } \\
\text { sleep apnea }\end{array}$} & \multirow{2}{*}{$\begin{array}{l}\text { At high risk } \\
\text { for OSA }\end{array}$} & \multirow{2}{*}{$\begin{array}{c}\text { At high risk for OSA } \\
\text { and no reported } \\
\text { sleep laboratory } \\
\text { testing referral }\end{array}$} & \multicolumn{2}{|c|}{$\begin{array}{c}\text { Prevalence rate ratio for sleep } \\
\text { laboratory testing referral* }(95 \% \mathrm{Cl})\end{array}$} \\
\hline & & & & & Unadjusted & Adjusted $\ddagger$ \\
\hline Canada & $4.8(4.1-5.5)$ & $3.4(2.8-4.0)$ & $19.0(17.6-20.4)$ & $18.0(16.6-19.4)$ & - & - \\
\hline Ontario & $7.7(6.3-9.2)$ & $4.6(3.4-5.8)$ & $18.3(15.9-20.8)$ & $17.1(14.7-19.5)$ & 1.0 & 1.0 \\
\hline British Columbia & $3.9(2.1-5.7)^{\S}$ & $3.8(2.2-5.3)^{\S}$ & $16.9(13.8-20.1)$ & $16.1(12.9-19.2)$ & $0.5(0.3-0.9)$ & $0.6(0.4-1.1)$ \\
\hline Prairies $\pi$ & $3.4(1.8-4.9)^{\S}$ & $2.5(1.4-3.6)^{\S}$ & $20.0(16.8-23.2)$ & $18.7(15.6-21.9)$ & $0.4(0.3-0.7)$ & $0.4(0.3-0.7)$ \\
\hline Quebec & $2.0(1.2-2.8)^{\S}$ & $1.9(1.0-2.7)^{\S}$ & $19.1(16.0-22.2)$ & $18.7(15.6-21.8)$ & $0.3(0.2-0.4)$ & $0.3(0.2-0.5)$ \\
\hline Atlantic*** & $3.4(1.4-5.3)^{\S}$ & $3.3(1.7-4.8)^{\S}$ & $23.7(20.7-26.6)$ & $22.6(19.6-25.6)$ & $0.4(0.2-0.8)$ & $0.3(0.2-0.6)$ \\
\hline
\end{tabular}

Bolded values indicate $P<0.05,1.0$ represents the reference category. ${ }^{*}$ Estimates weighted to the Canadian population; ${ }^{+}$High risk for OSA excludes individuals who reported diagnosed sleep apnea and is defined by the STOP tool; ${ }^{\ddagger}$ Adjusted for sex, age, ethnicity, body mass index, smoking, marital status, educational attainment, household income, chronic conditions and regular medical doctor; §Interpret with caution, estimate associated with high sampling variability; \/ncludes Alberta, Saskatchewan and Manitoba; **Includes New Brunswick, Nova Scotia, Prince Edward Island, and Newfoundland and Labrador

TABLE 7

Number of overnight sleep laboratories and number of sleep testing beds according to province and region, Canada, 2004 and 2010

\begin{tabular}{|c|c|c|c|c|c|c|c|c|}
\hline & \multicolumn{4}{|c|}{2004 (Flemons et al [17]) } & \multicolumn{4}{|c|}{ 2010* } \\
\hline & Sleep & & & & Sleep & & & \\
\hline & laboratories, $\mathbf{n}$ & Beds, $n$ & Population & Beds $/ 100,000$ & laboratories, $\mathbf{n}$ & Beds, $\mathbf{n}$ & Population ${ }^{\dagger}$ & Beds $/ 100,000$ \\
\hline Canada & 100 & 440 & $31,413,990$ & 1.4 & 131 & 698 & $34,108,800$ & 2.0 \\
\hline British Columbia & 5 & 18 & $4,141,272$ & 0.4 & 8 & 31 & $4,531,000$ & 0.7 \\
\hline Prairies & 9 & 24 & $5,276,242$ & 0.5 & 9 & 44 & $6,001,900$ & 0.7 \\
\hline Alberta & 5 & 14 & $3.113,586$ & 0.4 & 6 & 24 & $3,720,900$ & 0.6 \\
\hline Saskatchewan & 2 & 6 & $1,011,808$ & 0.6 & 2 & 10 & $1,045,600$ & 1.0 \\
\hline Manitoba & 2 & 4 & $1,150,848$ & 0.3 & 1 & 10 & $1,235,400$ & 0.8 \\
\hline Ontario & 69 & 340 & $12,068,301$ & 2.8 & 87 & 537 & $13,210,700$ & 4.1 \\
\hline Quebec & 12 & 44 & $7,455,208$ & 0.6 & 24 & 76 & $7,907,400$ & 1.0 \\
\hline Atlantic & 4 & 11 & $2,372,925$ & 0.5 & 3 & 10 & $2,346,300$ & 0.4 \\
\hline New Brunswick & 1 & 3 & 756,652 & 0.4 & 1 & 4 & 751,800 & 0.5 \\
\hline Nova Scotia & 2 & 6 & 944,765 & 0.6 & 2 & 6 & 942,500 & 0.6 \\
\hline Prince Edward Island & 0 & 0 & 139,913 & 0.0 & 0 & 0 & 142,300 & 0.0 \\
\hline Newfoundland and Labrador & 1 & 2 & 531,595 & 0.4 & 0 & 0 & 509,700 & 0.0 \\
\hline Territories & 0 & 0 & 100,042 & 0.0 & 0 & 0 & 111,500 & 0.0 \\
\hline Yukon & 0 & 0 & 29,924 & 0.0 & 0 & 0 & 34,500 & 0.0 \\
\hline Northwest Territories & 0 & 0 & 41,403 & 0.0 & 0 & 0 & 43,800 & 0.0 \\
\hline Nunavut & 0 & 0 & 28,715 & 0.0 & 0 & 0 & 33,200 & 0.0 \\
\hline
\end{tabular}

*Estimates do not include level 3 or pediatric testing facilities or data for the Territories; †Statistics Canada, 2010 (18)

high risk for OSA to diagnosed sleep apnea was largest in Quebec $(10: 1)$ and smallest in Ontario (4:1); the ratio for Canada overall was $6: 1$.

Assuming that the estimated 698 sleep laboratory beds in Canada operate for 365 days of the year, current sleep laboratories can perform a maximum of 254,770 tests per year. If one conservatively assumes that only one of four individuals at high risk for OSA requires a sleep laboratory test, we can estimate, using a more reasonable testing capacity estimate $(5$ days/week $\times 50$ weeks/year $\times$ operational capacity of $80 \%$ ), that it would take nearly eight years to test these individuals. This estimate does not take into account treatment follow-up requirements for sleep apnea or other sleep disorders, nor do they take into account testing requirements for children with sleep apnea and other sleep disorders.

Recent Canadian guidelines recommend that portable sleep monitoring studies can be used to confirm the diagnosis of OSA and institute appropriate treatment in patients with a moderate to high pretest probability of OSA (19). As such, portable monitoring is likely a viable and cost-effective option for increasing access to sleep testing in
Canada. However, portable sleep monitoring has limited application in patients with medical or psychiatric comorbid diseases as well as for the diagnosis of other forms of sleep apnea. In the majority of these patients, level-1 overnight sleep testing is required. Moreover, patients with obesity-hypoventilation currently require overnight PSG in a sleep laboratory for treatment to be initiated. Thus, even if portable monitoring becomes more widely available, the need for overnight sleep laboratory facilities is still apparent, particularly when one considers the trend of increasing obesity prevalence (and associated comorbidities). OSA management pathways should focus on early identification at the primary care level, with proper risk stratification and triaging to either in-laboratory PSG or home portable monitoring with management based on a validated ambulatory pathway protocol.

The STOP tool was validated in an older, preoperative population with higher average BMI measures as compared with the general population (16). Thus, our use of the tool for estimating the prevalence of high risk for OSA may have limited generalizability to the Canadian population. Furthermore, by using male-oriented OSA symptom criteria (eg, snoring), the tool may have been biased toward 
male selection. Women may present with different OSA symptoms compared with men, and are more likely to report insomnia, fatigue, to have hypothyroidism, to be treated for depression and be more overweight than men $(20,21)$.

The SARR questionnaire asked individuals if they had 'diagnosed sleep apnea' and, as such, we have no information regarding the type of sleep apnea. In our interpretation of the data, we assumed that the majority of these individuals had OSA. A few studies have demonstrated that the prevalence of central sleep apnea among individuals referred for sleep laboratory tests is $<5 \%(22,23)$.

In the majority of provinces, sleep laboratory tests are covered by provincial heath plans; however, in some (eg, Alberta, British Columbia, Nova Scotia and Quebec), privately funded sleep laboratories exist. We included privately funded sleep laboratories in our sleep laboratory survey recognizing that, for many Canadians, the costs of such testing constitutes a significant barrier to access.

\section{REFERENCES}

1. Lindberg E. Epidemiology of OSA. Eur Respir Mon 2010;50:51-68.

2. Sleep Apnea Rapid Response Survey, The Canadian Community Health Survey (2009a), Statistics Canada. Centre for Chronic Disease Prevention, Public Health Agency of Canada <www.statcan.gc.ca/daily-quotidien/091208/dq091208d-eng.htm> (Accessed July 2012).

3. The Canadian Community Health Survey (2009b), Statistics Canada. Centre for Chronic Disease Prevention, Public Health Agency of Canada. <www.statcan.gc.ca/cgi-bin/imdb/p2SV.pl?Funct ion $=$ get Survey $\&$ SDDS $=3226 \&$ lang $=e n \& d b=i m d b \& a d m=8 \& d i$ $s=2>$ (Accessed July 2012).

4. Bonsignore MR, Battaglia S, Zito A, Lombardi C, Parati G. Sleep apnoea and systemic hypertension. Eur Respir Mon 2010;50:150-73.

5. Grote L, Sommermeyer D. Early atherosclerosis and cardiovascular events. Eur Respir Mon 2010;50:174-88.

6. Mwenge GB, Rodenstein D. Public health and legal implications of OSA. Eur Respir Mon 2010;50:216-24.

7. Giles TL, Lasserson TJ, Smith B, White J, Wright JJ, Cates CJ. Continuous positive airways pressure for obstructive sleep apnoea in adults. Cochrane Database Syst Rev 2006(3):CD001106.

8. Kushida CA, Berry RB, Blau A, et al. Positive airway pressure initiation: A randomized controlled trial to assess the impact of therapy mode and titration process on efficacy, adherence, and outcomes. Sleep 2011;34:1083-92.

9. Marshall NS, Wilsmore BR, McEnvoy RD, Wheatley JR, Dodd MJ, Grunstein RR. Polysomnography in Australia - trends in provision. J Clin Sleep Med 2007;3:281-4.

10. Banno K, Walld R, Kryger MH. Increasing obesity trends in patients with sleep-disordered breathing referred to a sleep disorders center. J Clin Sleep Med 2005;1:364-6.

11. Tachibana N, Ayas NT, White DP. A quantitative assessment of sleep laboratory activity in the United states. J Clin Sleep Med 2005;1:23-6.

12. Flemons WW, Littner MR, Rowley JA, et al. Home diagnosis of sleep apnea: A systematic review of the literature: An evidence
Of the estimated 5.4 million Canadian adults who have either been diagnosed with sleep apnea or who are estimated to be at high risk for OSA, 4.5 million did not report having been referred for sleep laboratory testing. Canada currently has 131 diagnostic sleep laboratories, which is inadequate given the number of individuals at high risk for OSA, and the proportion of these individuals who would potentially benefit from testing and appropriate treatment.

AUTHOR CONTRIBUTIONS: J Evans performed the data analysis and contributed to interpretation and writing of the manuscript; C Rusu performed data quality review and contributed to data analysis; R Skomro and HS Driver contributed to data interpretation and writing of the manuscript; B Graham, I Mayers, J Reisman, T To and J Fleetham contributed to data interpretation and reviewing of the manuscript; L McRae contributed to survey design and implementation and review of the manuscript.

review cosponsored by the American Academy of Sleep Medicine, the American College of Chest Physicians, and the American Thoracic Society. Chest 2003;124:1543-79.

13. Rust KF, Rao JNK. Variance estimation for complex surveys using replication techniques. Stat Methods Med Res 1996;5:281-310.

14. Maldonado G, Greenland S. Simulation study of confounderselection strategies. Am J Epidemiol 1993;138:1102-9.

15. Hair JF Jr, Anderson RE, Tatham RL, Black WC. Multivariate Data Analysis, 3rd edn. New York: Macmillan, 1995.

16. Chung F, Yegneswaran B, Liao P, et al. STOP questionnaire: A tool to screen patients for obstructive sleep apnea. Anesthesiology 2008;108:812-21.

17. Flemons WW, Douglas NJ, Kuna ST, Rodenstein DO, Wheatley J. Access to diagnosis and treatment of patients with suspected sleep apnea. Am J Respir Crit Care Med 2004;169:668-72.

18. Estimates of population, by age group and for July 1, Canada, provinces and territories (2010), Statistics Canada. CANSIM table 051-0001 <www5.statcan.gc.ca/cansim/a26?lang=eng\& retrLang=en g\&id $=0510001 \& p a S e r=\& p a t t e r n=\& s t B y V a l=1 \& p 1=1 \& p 2=-$ $1 \&$ tabMode $=$ dataTable\&csid $=>$ (Accessed July 2012).

19. Fleetham J, Ayas N, Bradley D, et al. Canadian Thoracic Society 2011 guideline update: Diagnosis and treatment of sleep disordered breathing. Can Respir J 2011;18:25-47.

20. Young T, Hutton R, Finn L, Badr S, Palta M. The gender bias in sleep apnea diagnosis: Are women missed because they have different symptoms? Arch Intern Med 1996;156:2445-51.

21. Banno K, Kryger MH. The circuitous route to diagnosing sleep disorders in women: Healthcare utilization and benefits of improved awareness for sleep disorders. Sleep Med Clin 2008;3:133-40.

22. Javaheri S. Central sleep apnea. Clin Chest Med 2010;31:235-48.

23. Morgenthaler TI, Kagramanov V, Hanak V, Decker PA. Complex sleep apnea syndrome: Is it a unique clinical syndrome? Sleep 2006;29:1203-9. 


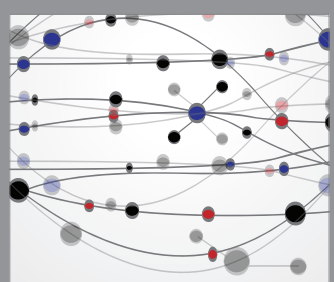

The Scientific World Journal
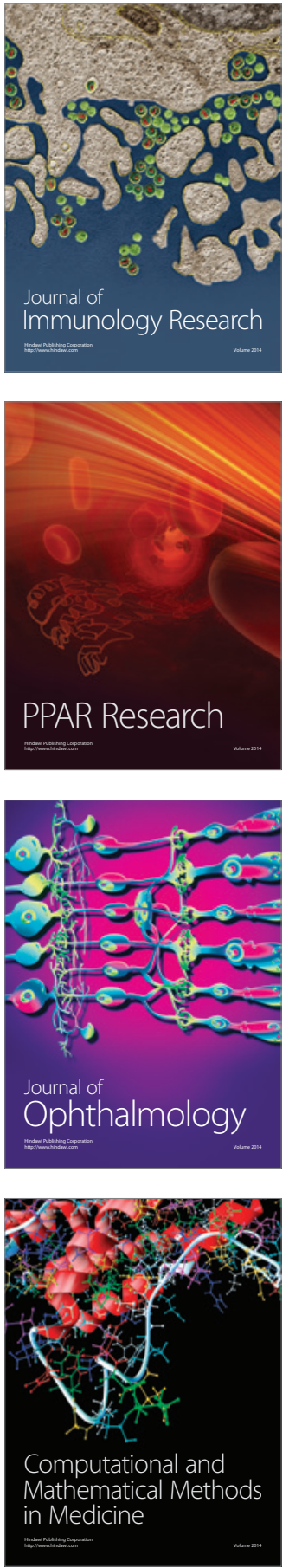

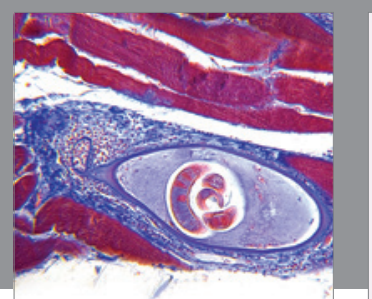

Gastroenterology Research and Practice

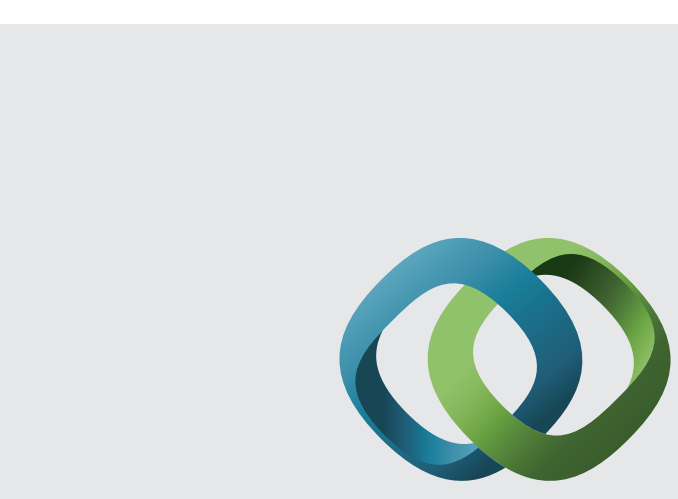

\section{Hindawi}

Submit your manuscripts at

http://www.hindawi.com
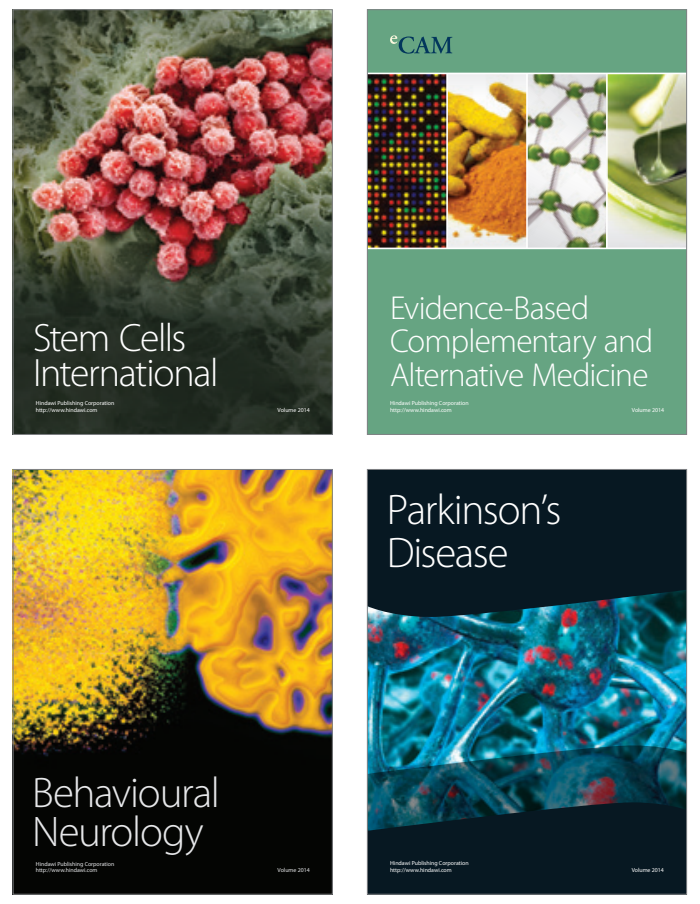
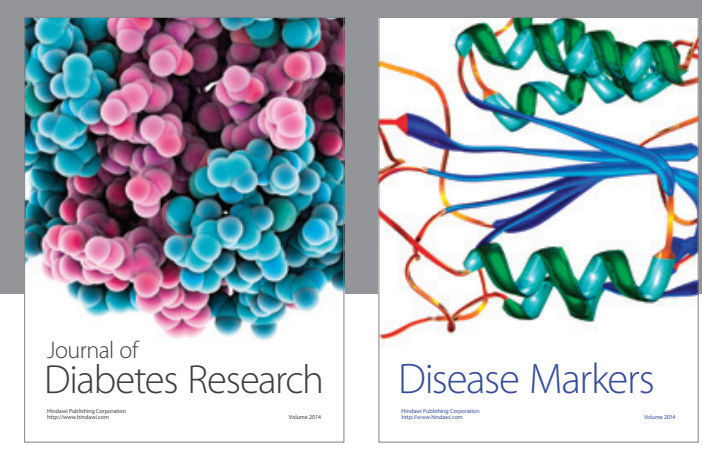

Disease Markers
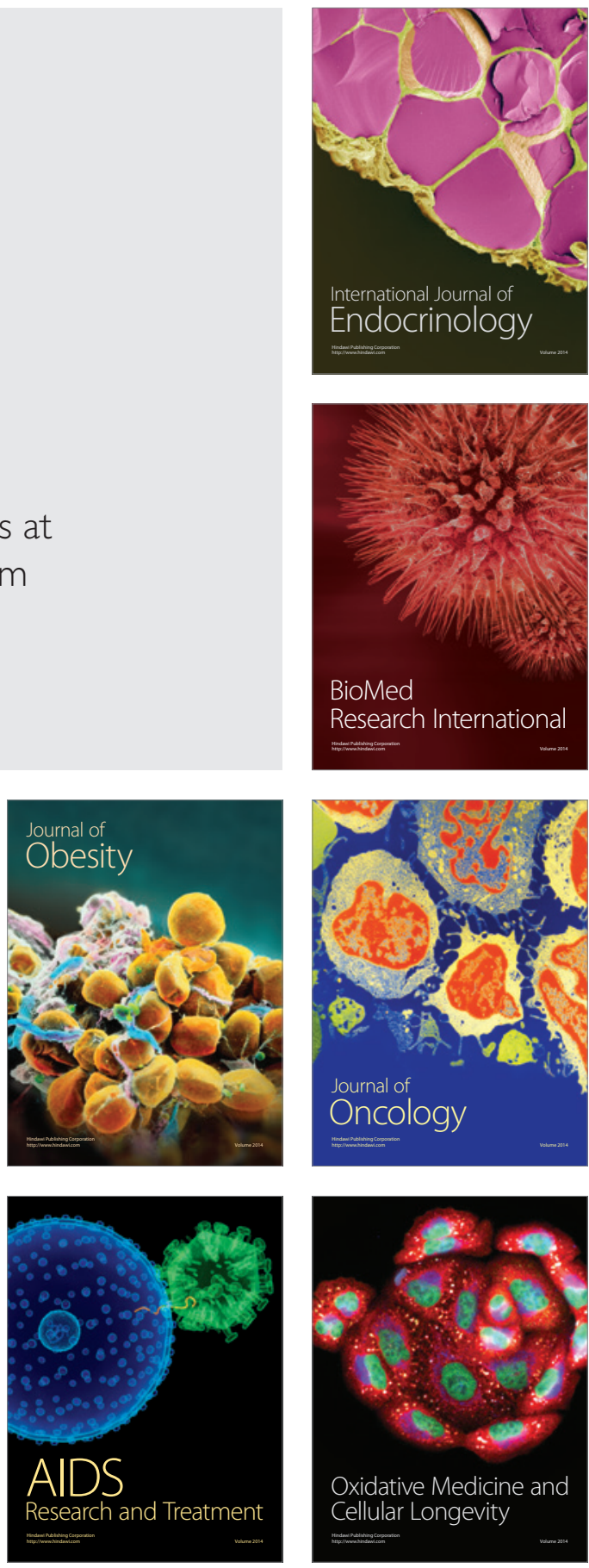